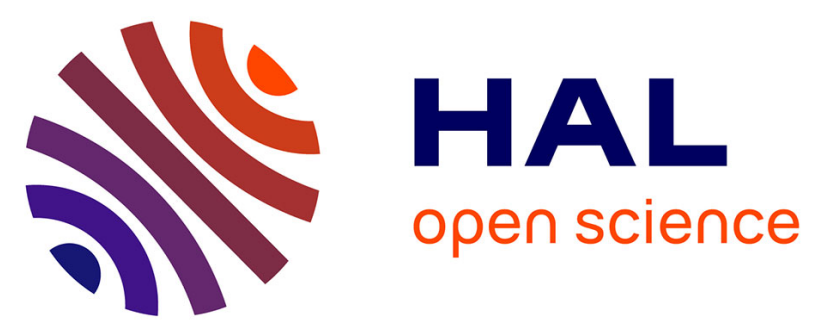

\title{
Les simulacres de la liberté?: le rôle du tacitisme dans les débats politiques de la fin du XVIIIe siècle (1696-1699)
}

Charles-Edouard - Edouard Levillain

\section{- To cite this version:}

Charles-Edouard - Edouard Levillain. Les simulacres de la liberté?: le rôle du tacitisme dans les débats politiques de la fin du XVIIIe siècle (1696-1699). XVII-XVIII Revue de la Société d'études anglo-américaines des XVIIe et XVIIIe siècles , 2005, 60, pp.143 - 154. 10.3406/xvii.2005.2024 . hal-03251137

\section{HAL Id: hal-03251137 \\ https://hal.science/hal-03251137}

Submitted on 6 Jun 2021

HAL is a multi-disciplinary open access archive for the deposit and dissemination of scientific research documents, whether they are published or not. The documents may come from teaching and research institutions in France or abroad, or from public or private research centers.
L'archive ouverte pluridisciplinaire HAL, est destinée au dépôt et à la diffusion de documents scientifiques de niveau recherche, publiés ou non, émanant des établissements d'enseignement et de recherche français ou étrangers, des laboratoires publics ou privés. 


\section{Les simulacres de la liberté ? : le rôle du tacitisme dans les débats politiques de la fin du XVIIle siècle (1696-1699)}

\section{Charles-Edouard Levillain}

\section{Abstract}

The purpose of this essay is to look at the influence of Tacitism on the political debates of the late 1690s (1696-1699). Far from receding after the 1650s, the use of Tacitus's historical works played a decisive role in the way some Englishmen perceived the shifts of William III's career and the nature of the regime born out of the Glorious Revolution settlement.

\section{Résumé}

L'objet de cet essai est d'analyser l'influence du tacitisme sur les débats politiques de la fin des années 1690 (1696-1699). La principale thèse de l'article est que, loin de reculer après les années 1650, l'utilisation des cuuvres historiques de Tacite joua un rôle décisif dans la façon dont certains Anglais perçurent les évolutions de la carrière de Guillaume III et la nature du régime issu de la Glorieuse Révolution.

\section{Citer ce document / Cite this document :}

Levillain Charles-Edouard. Les simulacres de la liberté ? : le rôle du tacitisme dans les débats politiques de la fin du XVIIle siècle (1696-1699). In: XVII-XVIII. Revue de la société d'études anglo-américaines des XVIle et XVIIle siècles. N60, 2005. pp. 143-154.

doi : 10.3406/xvii.2005.2024

http://www.persee.fr/doc/xvii_0291-3798_2005_num_60_1_2024

Document généré le 29/09/2015 


\title{
LES SIMULACRES DE LA LIBERTÉ? \\ LE RÔLE DU TACITISME DANS LES DÉBATS \\ POLITIQUES DE LA FIN DU XVIIIe SIÈCLE (1696-1699)
}

\begin{abstract}
Résumé: L'objet de cet essai est d'analyser l'influence du tacitisme sur les débats politiques de la fin des années 1690 (1696-1699). La principale thèse de l'article est que, loin de reculer après les années 1650, l'utilisation des œuvres historiques de Tacite joua un rôle décisif dans la façon dont certains Anglais perçurent les évolutions de la carrière de Guillaume III et la nature du régime issu de la Glorieuse Révolution.
\end{abstract}

Abstract: The purpose of this essay is to look at the influence of Tacitism on the political debates of the late 1690s (1696-1699). Far from receding after the $1650 \mathrm{~s}$, the use of Tacitus's historical works played a decisive role in the way some Englishmen perceived the shifts of William III's career and the nature of the regime born out of the Glorious Revolution settlement.

Fidèle au thème de ce colloque, je me propose de réfléchir à la manière dont l'œuvre historique de Tacite a été utilisée dans l'Angleterre de la fin du XVIIe siècle à des fins de propagande. Le titre "les simulacres de la liberté" renvoie de façon quelque peu provocatrice à une formule classique de Tacite décrivant l'action politique de certains empereurs romains, 'formule que nous appliquerons ici à la fin du règne de Guillaume III et, plus précisément, aux débats politiques qui eurent cours entre 1696, date d'une tentative avortée d'assassinat contre le roi, et 1699 , date de la fin des débats relatifs à l'armée de métier du roi.

L'idée de ce sujet peut se justifier de deux façons. D'abord, le règne de Guillaume III a assez récemment fait l'objet d'une redécouverte après

1. Tacite, Annales, 1: 72, 3. Tacite parle ici de Tibère. Pour les traductions françaises de Tacite, nous utiliserons l'édition suivante: Tacite, Euvres complètes, trad. Pierre Grimal (Paris: Gallimard, 1990). 
de longues années de désintérêt, pour ne pas dire de silence. ${ }^{2}$ Les remises en cause de l'historiographie whig ont eu des conséquences majeures sur les études guillaumiennes. Le règne de Guillaume III est désormais abordé dans une perspective anglo-hollandaise, et non plus dans une perspective étroitement britannique. ${ }^{3}$ On ne saurait oublier en effet, que, entre 1689 et 1702, Guillaume III fut à la fois roi d'Angleterre, d'Écosse et d'Irlande, et stathouder des Provinces-Unies. Ensuite, l'historiographic du tacitisme appelle des ajustements. L'essentiel des études consacrées au tacitisme à l'époque moderne considèrent qu'il connut une heure de gloire entre la fin du XVIe siècle et le milieu du XVIIe siècle, avant de décliner à partir des années 1650$1660 .^{4} \mathrm{Si}$ on compare le nombre de traductions et de commentaires publiés avant et après le milieu du XVIIe siècle, cette thèse est tout à fait défendable. Néanmoins, tout dépend de la façon dont s'entend la notion de tacitisme. Les traductions et les commentaires de Tacite ne constituèrent en réalité qu'une composante du tacitisme, n'en épuisant ni le sens ni l'influence. Il faut donc voir dans le tacitisme une réutilisation large, diffuse et parfois contradictoire de l'œuvre historique de Tacite, tant pour comprendre le présent à la lumière du passé que pour faire œuvre de propagande dans un contexte politique donné.

Ce cadre posé, il paraît nécessaire d'affiner la définition du problème. C'est chose connue: la Glorieuse Révolution a été soutenue par un immense travail de propagande, tant du côté anglais que du côté hollandais, visant à opposer les libertés protestantes défendues par Guillaume IIl à la prétendue tyrannie louis-quatorzienne et à la supposée collusion de Jacques II avec cette même tyrannie. Guillaume III, en somme, était du côté de la liberté; Louis XIV et Jacques II, en revanche, du côté du "papisme et du gouvernement arbitraire" ("popery and arbitrary government"). La recherche récente a permis de rappeler que le règne de Guillaume III avait été émaillé de graves crises, à cause, essentiellement, d'une dérive belliciste qui, aux yeux d'une fraction de l'opinion publique, constituait une menace pour les libertés publiques. ${ }^{5}$

2. Voir Craig Rose, England in the 1690s (London: Blackwell, 1999).

3. Voir David Onnekink, The Anglo-Dutch Favourite. The Career of Hans Willem Bentinck, 1st Earl of Portland (1649-1709) (thèse inédite de l'université d'Utrecht, 2004).

4. Kenneth C. Schellhase, Tacitus in Renaissance Political Thought (Chicago: the U of Chicago P, 1976) 165.

5. Voir Julian Hoppit, A Land of Liberty? England 1689-1727 (Oxford: Clarendon, 2000). 
Parallèlement, l'espoir d'une restauration de Jacques II restait très vif chez les Jacobites et chez certains Tories, laissant planer une réelle menace sur la légitimité et la stabilité du régime guillaumien. Enfin, il faut admettre que Guillaume III fut un roi mal-aimé, pcut-être mal compris, écartelé entre un dessein européen dicté par sa lutte quasiobsessionnelle contre Louis XIV, et un destin anglo-hollandais bien difficile à assumer dans la diversité de ses exigences. Mon analyse portera sur la façon dont la carrière militaire et politique de Guillaume III a été interprétée à la lumière de l'histoire romaine et de l'œuvre de Tacite. Mon principal objectif est de montrer qu'il existait à la fin du XVIIe siècle un espace culturel et idćologique anglo-hollandais dont la richesse et la fécondité transparaissent avec force à travers les relectures parallèles et croisées de Tacite.

À l'époque moderne, le tacitisme se définissait avant tout par son caractère transfrontalier. L'Europe était alors constituée de pays aux régimes politiques et religieux différents, mais la diversité de ces régimes politiques et religieux était transcendée par ce que l'on appelait depuis le XVe siècle la "république des Lettres," autrement dit un espace de circulation des idées qui ignorait les séparations imposées par les frontières et les divisions imposées par les doctrines politiques et religieuses. ${ }^{6}$ C'est ainsi que la renaissance du tacitisme à la fin du XVIe siècle fut avant tout un phénomène européen qui partit de Hollande pour se diffuser à travers tout le continent. ${ }^{7}$ L'une des figures marquantes de ce renouveau fut Juste Lipse, dont la traduction et le commentaire de Tacite connurent aussitôt un succès considérable. La préface de la traduction anglaise de 1581 méritc d'être citée:

Look well! he presents kings and monarchs to you, in a word, the theatre of our life today... I I find the ways and means of destroying liberty; I find the ill-fated efforts to recover lost liberty. I read in turn of tyrants overthrown and laid down; I read of power insecure when wielded to excess. ${ }^{8}$

6. Pour ce sujet, voir Paul Dibon, "Communication in the Respublica Literaria of the Seventeenth Century," Respublica Literarum. Studies in the Classical Tradition, I, (1978): 43-55 et Johannes Alphonsus Henricus G. Bots, De Republiek der Letteren. Ideaal en Werkelijkheid (Amsterdam: Amsterdam UP, 1997).

7. Stricto sensu, la Hollande désignait l'une des sept provinces des Provinces-Unies issue de l'Union d'Utrecht de 1579. Lato sensu, elle peut servir à désigner l'ensemble des ces territoires.

8. Extrait cité in Torrey, Tacitus and the Tacitean Tradition, ed. James Luce et Anthony John Woodman (Princeton: Princeton UP, 1993) 138. 
Le parallèle avec la préface de Henry Savile à sa traduction de Tacite (1598) est frappant:

In these four books of the story thou shall see all the miseries of a torn and declining state: the Empire usurped; the princes murdered; the people wavering; the soldiers tumultuous. . . If thou mislike their wars be thankful for thine own peace; if thou abhor their tyrannies, love and reverence thine own life, just, and excellent Prince. If thou doest detest their anarchy, acknowledge our own happy government. ${ }^{9}$

Chacun à leur façon, Juste Lipse et Henry Savile étaient fascinés par le caractère intemporel de l'cuuvre de Tacite, à savoir le sentiment qu'elle constituait un réservoir de maximes et de réflexions qui pouvaient aisément s'appliquer au temps présent. Mais intemporel nc veut pas dire anhistorique. Il serait erroné de penser que les hommes des XVIe et XVIIe siècles dépouillèrent l'œuvre de Tacite de son caractère historique pour la transformer en une sorte d'abrégé de principes politiques destiné à éclairer la conduite des princes. Les commentaires de l'époque juxtaposaient deux niveaux de lecture. Il s'agissait à la fois de s'interroger sur le mouvement de l'histoire, notamment sur sa faculté apparente de répétition, et sur les fondements du bon gouvernement, et cela dans une perspective plus morale et philosophique.

Cette remarque appelle une mise au point importante sur Tacite. Tous les spécialistes de Tacite s'accordent à dire que Tacite n'était pas un philosophe, encore moins un théoricien du politique, mais un observateur "des mœurs et des hommes" (mores virique), ${ }^{10}$ qui mettait sa plume d'historien au service d'une enquête approfondie sur les "secrets du pouvoir" (arcana imperii)," formule célèbre que l'on retrouve fréquemment dans la littérature politique des années $1690 .^{12}$ Les "mystères de l'État," tels que Tacite les analyse, restaient d'autant plus mystérieux qu'il ne prend jamais position, ou jamais de façon assez claire pour permettre à ses lecteurs d'en faire un ennemi de la république

9. Henry Savile, preface, The End of Nero and Beginning of Galha. Four Books of the Histories of Cornelius Tacitus (Oxford, 1591). Pour plus de détails sur les enjeux politiques et idéologiques de cette traduction, voir David Womersley, "Sir Henry Savile's translation of Tacitus and the political interpretation of Elizabethan texts," The Review of English Studies, 42.167 (1991): 313-342.

10. Ronald Syme, Tacitus (Oxford: Oxford UP, 1958) 2: 550, et Ronald Mellor, Tacitus (London: Routledge, 1993) 50.

11. Tacite, Annales 2: 361 .

12. Voir Algernon Sidney, Discourses concerning government (London, 1698) 8. 
ou de l'empire. Comme l'a expliqué Ronald Syme, Tacite était l'historien "de la voie médiane,"13 croyant à une réconciliation entre l'esprit républicain et l'idéal d'un régime impérial protégé de la corruption et de la tyrannie. Autrement dit, Nerva ou Trajan plutôt que Tibère ou Néron. Ainsi s'explique l'attachement de Tacite à la vertu, seule capable, selon lui, de maintenir le pouvoir personnel d'un prince à l'intérieur des bornes de la loi.

L'ambiguïté de Tacite à l'égard de la République et du Principat permet de comprendre pourquoi son ouvre a été interprétée de façon contradictoire et pourquoi un même passage a été utilisé pour dire une chose et son contraire. Tout, en somme, était une question de perspective, preuve, s'il en était besoin, que le tacitisme était marqué par une très grande flexibilité qui en faisait un instrument privilégié de propagande. Il existait dans l'Angleterre et la Hollande de la fin du XVIIe siècle trois manières de percevoir et de comprendre le glissement de la République au Principat tel qu'il est décrit par Tacite, notamment dans l'ouverture des Annales.

La ville de Rome au commencement appartint à des rois; la liberté et le consulat furent établis par Lucius Brutus. La dictature était conférée chaque fois pour un temps limité. . . Ni Cinna ni Sulla ne conservèrent longtemps le pouvoir absolu; la prépondérance de Pompée et de Crassus passa bientôt à César, la puissance militaire de Lépide et d'Antoine à Auguste, qui avec le titre de prince reçut sous son autorité absolue l'ensemble de l'État, épuisé par les guerre civiles. ${ }^{14}$

La première interprétation était républicaine et anti-monarchique. On la devait à Hugo Grotius qui avait publié en 1610 un ouvrage intitulé Liber de Antiquitate Reipublicae Batavicae, dédié aux États de Hollande et traduit en anglais on 1649 sous le titre $A$ Treatise of the Antiquity of the Commonwealth of the Batavers, which is now the Hollanders. Pour Grotius, la notion de liberté était incompatible avec un régime monarchique, qui était intrinsèquement contraire au tempérament politique des Bataves. Défenseur acharné de la souveraineté des États provinciaux face au pouvoir grandissant du stathoudérat, Grotius inscrivait les libertés bataves dans la continuité des libertés que Tacite prêtait aux tribus germaniques dans son De Germania: "Les rois, d'ailleurs, n'ont pas un pouvoir illimité ni arbitraire et les chefs, par

13. Syme, 2: 548.

14. Tacite, Annales 1: 1-8. 
l'exemple qu'ils donnent plutôt que par le pouvoir dont ils sont investis ... doivent leur autorité à l'admiration qu'ils provoquent." ${ }^{15}$

À cette première interprétation républicaine et anti-monarchique s'opposait une conception orangiste des rapports entre république et principat, liberté et pouvoir personnel. La plus importante est due à l'érudit Marcus Boxhorn, ${ }^{16}$ lequel publia en 1643 une édition annotéc des cuvres de Tacite dont on trouve une trace dans plusieurs bibliothèques de figures intellectuelles de l'Angleterre de la fin du XVIIe siècle, et notamment dans celle de Matthew Prior, poète et diplomate qui fut stipendié par la Cour en 1697-9 pour répondre aux attaques contre l'armée de métier de Guillaume III. Boxhorn interprétait l'incipit des Annales de la manière suivante:

Ceux qui estiment que Tacite oppose ici la liberté au pouvoir des rois vont au-delà de ce que dit le texte et ne comprennent pas ce que l'auteur a voulu dire. Car dans quelque empire que ce soit, même dans un Principat, il y a de la liberté si le régime en question reste dans ses justes bornes. ${ }^{17}$

Boxhorn reprit cette idée dans un texte latin publié en 1651, un an après l'exclusion des Orange-Nassau des hautes charges de l'État (1650), faisant sienne une autre formule célèbre de Tacite à propos de l'avènement d'Auguste: "Il n'y avait pour guérir les discordes de la patrie

15. Hugo Grotius, The Antiquity of the Batavian Republic, trad. Jan Waszink (Assen: Van Gorcum, 2000) 57-59. Pour la citation originale de Tacite, voir De Germania 7: 1. On notera qu'Algernon Sidney faisait aussi du mode de gouvernement des Germains le modèle originel du régime politique de l'Angleterre: "This [Gothic polity] is above all seen in the German nations, from whom we draw our original and government," Discourses concerning government (1698). Citation donnée in Ronald Mellor, Tacitus: the Classical Heritage (London: Garland, 1995) 127.

16. Le manuscrit se trouve à Londres (BL, ADD MSS 70 362, fol. 22). Pour défendre la cause de Guillaume III, Prior rédigea un libelle intitulé $A$ New Answer to An Argument against a Standing Army (London, 1697). On trouve aussi une trace d'une édition de 1664 du Tacite de Boxhorn dans la bibliothèque privée de troisième comte de Shaftesbury, cheville ouvrière de la faction républicaine du Nouveau Parti du Pays (London, National Archives [NA], Shaftesbury Papers, PRO 30/24/23/10, fol. 76).

17. "Qui statuunt libertatem et regium imperium opponi hoc loco a Tacito. excedunt, neque capiunt auctoris mentem. Nam in quovis imperio, etiam in principatu, est libertas, si is quidem recte se habeat." Caii Cornelii Taciti quae exstant M.Z Boxhornius recensuit et animadversionibus nonnullis illustravit (Amsterdam, 1643) 3. Boxhorn s'inspire directement du De Agricola 3: 1, où Tacite fait référence à Nerva. 
d'autre remède que de la soumettre au pouvoir d'un seul." ${ }^{18} \mathrm{C}^{\prime}$ est une idée qui prit tout son sens lors de l'invasion française de 1672 , lorsque les Provinces-Unies faillirent basculer dans le chaos et que Guillaume III fut nommé stathouder, capitaine-général et amiral-général pour sauver son pays du désastre militaire, et à nouveau en décembre 1688, lorsque l'Angleterre connut une vacance du pouvoir de quelques jours après le départ inopiné de Jacques II. Dans les deux cas, la propagande orangiste saisit l'occasion pour présenter Guillaume III comme un personnage providentiel qui permettait de restaurer l'ordre et l'unité de l'Etat et de conjurer le spectre de la guerre civile. ${ }^{19}$

L'avènement de Guillaume III sur le trône d'Angleterre en 1689 fit naître une troisième forme de tacitisme qui, elle, était d'inspiration jacobite. En 1698 fut publiée une nouvelle traduction annotée de Tacite à laquelle contribua en particulier John Dryden, poc̀te lauréat de la Restauration disgracié à la suite de la Glorieuse Révolution. Agrémentée de "notes historiques" et de "réflexions politiques," cette traduction est logiquement animée par la volonté de remettre en cause la légitimité du pouvoir de Guillaume III et de le présenter comme un usurpateur, un nouveau Cromwell, comme le répéta à l'envi la propagande jacobite. On peut citer ce refrain d'un poème de décembre 1690 intitulé The Weasel Uncased: "So let O.P or P.O be King / Or anyone else, it is the same thing." ${ }^{20}$ Dans le même esprit, on trouve le commentaire suivant à propos de l'ouverture des Annales: "Nothing is so weak and so obnoxious to a reverse of fortune, as a power which hath neither right nor reason for its foundation." Et plus loin: "Tacitus seems to observe here, that Rome was never at rest, after the expulsion of its Kings, until it return'd to the government of a single person, as to its first principle." ${ }^{21}$ De ce commentaire se dégagent deux idées: d'abord celle

18. Amsterdam, Universiteit Bibliotheek Amsterdam, Marcus Zuerius van Boxhorn, Dissertationes politicae de Romanorum imperio, (Amsterdam, 1651) 325. Pour la citation originale de Tacite, voir Annales 1: 9, 4.

19. Pour un exemple de pamphlet orangiste publić en 1672, l'année du désastre, voir Souverains antidotes contre les maux de la Hollande causés par le mauvais régime de la cabale et la faction de Loevestein (NA, State Papers [SP], 119/187). Pour un exemple de pamphlet publié en 1688, l'année de la Glorieuse Révolution, voir Address of William of Orange to the English fleet (BL, Egerton MSS, 1755, fol. 6).

20. Poems on Affairs of State, vol.5, ed. William James Cameron (New Haven: Yale UP, 1971) 250.

21. The Annals and History of Cornelius Tacitus. Made English by several Hands, vol.1 (London, 1698) bk. 1: 4. Pour une analyse de la traduction de Dryden et de son 
d'une supériorité quasi-ontologique des régimes monarchiques sur les régimes républicains (on retrouve là une trace de l'aversion profonde de Jacques II pour tout ce qui, de près ou de loin, rappelait la période de l'Interrègne); ensuite l'idée que la force d'un régime monarchique repose sur sa légitimitć. Lorsque l'on se souvient que plusieurs mois de débats furent nécessaires en 1689 pour décider du titre qui serait accordé à Guillaume III, ${ }^{22}$ on comprend que le commentaire de l'édition de 1698 avait des implications pratiques très claires: pour les Jacobites, il s'agissait de renverser un roi dont l'absence de légitimité favorisait les querelles intestines, affaiblissant la monarchie britannique de l'intérieur.

Après avoir défini ces trois courants de lecture de Tacite, il paraît maintenant utile de passer à une analyse plus précise des débats politiques des années 1696-1699. L'analyse sera concentrée sur deux thèmes: le premier sera celui des rapports entre tyrannie et usurpation; le second celui des rapports entre tyrannie et corruption.

Les rapports entre tyrannie et usurpation constituaient logiquement un thème de prédilection des Jacobites, qui ne manquaient aucune occasion d'insister sur le caractère frauduleux de la royauté de Guillaume III. Ce thème prit un relief particulier en 1696 lorsque le Roi échappa à une tentative d'assassinat. Du point de vue des Jacobites, l'incident fut considéré comme une tentative avortée de mise en pratique des principes posés dans Killing No Murder, célèbre pamphlet d'Edward Sexby publié en 1657, qu'avaient justement réédité les Jacobites en 1689. Killing No Murder constituait une défense du tyrannicide qui, après avoir été appliquée à Cromwell en 1657, l'était désormais à Guillaume III. On y trouve une critique très virulente de l'usurpation impériale, avec cette idée que les tyrans parviennent à leur fin bien plus par la ruse que par la force ("much more by fraud than force").

contexte politique, voir Steven Zwicker et David Bywaters, "Politics and Translation: the English Tacitus of 1698," HLQ 52.3 (1989): 319-346.

22. Guillaume III pensait que la Convention parlementaire de 1689 voulait réduire ses pouvoirs à celui du doge de Venise et s'en plaignit auprès de George Savile, marquis de Halifax. The Life and Letters of Sir George Savile, ed. Helen C. Foxcroft (London, 1898) 2: 203. Ambassadeur de Charles II d'Espagne à Londres, Don Pedro Ronquillo imaginait que Guillaume III se verrait attribuer un rôle de régent, à l'image du duc de Gloucester pendant la minorité d'Édouard VI (Bruxelles, Archives générales du royaume, Manuscrits divers, I.115, 950-951, Relacion y memorias historicas y politicas, fol. 420). 
Guillaume III était directement visé par cette attaque: "Almost all tyrants have been first captains and generals for the people, under pretences of vindicating or defending their liberties."23

Dans une telle perspective, l'accession de Guillaume III au titre de capitaine-général en 1672 avait donc été un premier pas dans la construction du régime tyrannique sous lequel vivait l'Angleterre depuis 1689. Il n'est pas étonnant de voir les auteurs de l'édition de 1689 invoquer Tacite pour corroborer leur thèse: "Pour abattre l'empire, ils mettent en avant la liberté; s'ils parviennent à l'abattre, ils s'en prendront aussi à la liberté elle-même." ${ }^{24}$ Les Jacobites se servaient donc de Tacite pour montrer que le slogan choisi par Guillaume III pour la campagne de 1688, "Pro religione et libertate," était en réalité un leurre, une manière habile de donner à la tyrannie le visage de la liberté. Ce qui surprend, c'est moins le recours par les Jacobites à ce thème que sa quasi-disparition dans la littérature républicaine anglaise des années 1690. Après tout, le thème de l'usurpation impériale occupait une place essentielle dans certains textes de Milton, tel le Pro Populo Anglicano Defensio (1651, rééd. 1692) ou encore dans les Court Maxims d'Algernon Sidney, texte rédigé par celui-ci au cours d'un séjour en Hollande au milieu des années $1660 .{ }^{25}$ La raison de ce silence est double: d'abord, le thème de l'usurpation impériale est devenu, dans les années 1690, l'un des chevaux de bataille de la propagande jacobite, ce qui, pour les républicains, aurait entrainé une confusion idéologique regrettable.

Il y avait une deuxième confusion dont il fallait à tout prix se garder: celle que le Roi pouvait opérer entre républicains anglais et hollandais. À partir du milieu des années 1670, la littérature républicaine batave avait repris à son compte le thème de l'usurpation impériale pour dénoncer la façon dont Guillaume III et son entourage manipulaient les asscmblées provinciales. ${ }^{26}$ Or, contrairement à leurs homologues bataves, anti-monarchiques, les républicains anglais défendaient l'idée d'un justc équilibre entre roi, Lords et Communes. La meillcure preuve en est cette expression de John Trenchard,

23. Killing No Murder (London, 1689) 6.

24. Killing No Murder 6. La citation est tirée des Annales 16: 22, 4.

25. John Milton, Pro Populo Anglicano Defensio, in The Works of John Milton, ed. Frank Allen Patterson (New York: Columbia UP, 1932) 7: 317. Algernon Sidney, Court Maxims, ed. Hans Blom, Eco Haitsma Mulier \& Ronald Jasse (Cambridge: Cambridge UP, 1996) 138-139. Milton et Sidney font tous deux référence à Tibère.

26. Some things concerning the prince of Orange worthy of great reflection, 1675? (NA, Williamson Papers, SP 9/202!1, fol. 220). 
figure de proue de la faction républicaine du Nouveau Parti du Pays et grand lecteur de Tacite: "As passionate as he was for liberty, he was not for a commonwealth in England. He neither believed it possible, nor wished for it. ${ }^{27}$ Comme le rappelait le républicain Robert Molesworth dans un texte publié au début des années 1710 , l'expérience du protectorat avait entaché la notion de "commonwealth" qui, jusque-là, désignait simplement un régime politique, sans apporter de précisions sur les modalités de son fonctionnement. ${ }^{28}$

Passons maintenant à un examen des rapports entre tyrannie et corruption. Une formule de John Trenchard peut servir à poser le problème. "The Roman virtue and the Roman liberty expired together. Tyranny and corruption came upon almost hand in hand." ${ }^{29}$ On reconnaît là l'un des thèmes favoris de Tacite, à savoir que le développement de la tyrannie allait de pair avec une corruption des mœurs, et notamment une corruption de "l'antique vertu" qui avait servi de fondement aux libertés romaines. ${ }^{30}$ D'où, inversement, son admiration pour de grandes figures du stoïcisme comme Caton l'Ancien, qui, selon lui, avait incarné la vertu romaine dans toute sa plénitude. Ce n'est donc pas un hasard si John Trenchard se fit surnommer "Cato". L'obsession de la Restauration fut ce que l'on appela "le papisme et le gouvernement arbitraire." La question du papisme fut évacuée par le fait que Guillaume III était calviniste, appartenant donc, aux yeux des anglicans, à une aile dure du protestantisme. En revanche, la question du gouvernement arbitraire subit une métamorphose, se voyant désormais associée à une inquiétude croissante à propos de la moralité publique, de la vertu individuelle et collective de la nation anglaise.

Cette préoccupation était une conséquence directe de la révolution financière, autrement dit des changements que la guerre de la Ligue d'Augsbourg (1688-1697) entraîna dans le fonctionnement de l'économie britannique. Allant à l'encontre d'une formule célèbre de Machiavel, ${ }^{31}$ l'économiste et politologue tory Charles Davenant notait en

27. John Trenchard, preface, Cato's Letters (London, 1724) 1: 42.

28. Robert Molesworth, The Principles of a Real Whig, contuined in a Preface to the famous Hotman's Franco-Gallia (London, 1755) 7.

29. John Trenchard, Cato's Letters 1: 208.

30. Alain Michel, Tacite et le destin de l'empire (Paris: Arthaud, 1966) 112.

31. "Dico pertanto, non l'oro, come grida la commune opinione, essere il nervo della guerra, ma i buoni soldati," Niccolò Machiavelli, Discorso sopra la prima deca di Tito-Livio, in Opere, ed. Sergio Bertelli (Milano, 2001) 1: 255. 
1695 que c'était l'argent et non les "bons soldats" qui constituait désormais le nerf de la guerre. ${ }^{32}$ Voilà pourquoi Davenant vitupérait une époque qui, à ses yeux, était gangrenée par le règne de l'argent et, conséquence inévitable, la corruption. La British Library conserve un manuscrit inédit de Davenant intitulé An Essay upon Public Virtue où l'auteur résumait sa pensée de façon claire: "When any Empire is destined to be undone, or to lose its freedom, the seeds of this ruin are to be first seen in the corruption of its manners, and from hence spring all the disorders in the state whatsoever." 33

Davenant s'en prenait en particulier à la corruption des moeurs politiques, mais aussi, thème éminemment tacitéen, à la corruption de l'ćloquence qui, selon lui, était l'un des leviers ayant permis aux chefs militaires de la fin de la République de précipiter l'extinction des libertés romaines. ${ }^{34}$ Lorsque John Somers, le chef de la junte Whig, était raillé pour son "éloquence plus que Romaine" dans un poème satirique de Sir Richard Blackmore en 1700, il subissait une attaque d'inspiration ouvertement tacitéenne. ${ }^{35}$ Ce qu'il faut retenir du discours tenu par ces protagonistes des débats politiques de la fin du XVIIe siècle, c'est leur volonté de revenir aux sources et aux origines idéales de la vertu britannique, tout comme Grotius s'était fait le chantre de la vertu des anciens Bataves. C'est le cas de Sir Richard Blackmore dans $A$ Satyr against Wit:

How happy were the unpolished times,

As our forefathers vigorous were and brave,

So they were virtuous, wise, discreet and grave,

Detesting both alike the wit and the knave. ${ }^{36}$

Ce phénomène peut s'expliquer par une inquiétude face aux changements très importants induits par la Glorieuse Révolution. Il n'est

32. Charles Davenant, An Essay upon the Ways and Means of supplying the War, (London, 1695) 27.

33. BL, Harleian MSS 1223, fol. 12. Manuscrit probablement rédigé au milieu des années 1690 .

34. BL, Harleian MSS 1223, fol. 27-28.

35. Sir Richard Blackmore, A Satyr against Wit (London, 1700) 7. Certaines études n'ont pas manqué de souligner la forte influence exercée sur ce texte par les Ragguagli di Parnasso (1612-1613) de Trajano Boccalini, cuvre traduite pour la première fois en anglais en 1622 et fortement influencée par Tacite. Richard Thomas, "Trajano Boccalini's Ragguagli di Parnasso and its Influence upon English Literature," Aherystwyth Studies 3 (1922): 91.

36. Blackmore 3. 
pas rare que le retour aux sources soit lié à un besoin de redéfinition des paramètres traditionnels du temps et des l'espace. Ce fut sans doute le cas dans les années 1690, à une époque où les îles britanniques glissaient vers ce que l'on a ensuite appelé la deuxième guerre de Cent Ans.

Bien plus, il est permis de voir dans la prégnance du tacitisme au cours des années 1696-1699 un démenti à l'idée répandue d'une domination du machiavélisme dans les débats politiques. ${ }^{37}$ Il ne s'agit pas de nier l'importance de Machiavel dans la construction du discours politique des années 1690, mais de reconnaître au tacitisme un rôle essentiel dans la mobilisation des esprits au cours des crises successives qui ponctuèrent le règne de Guillaume III. Revenir à Machiavel consistait, somme toute, à revenir aux sources romaines par le prisme d'un médiateur. Revenir à Tacite permettait à chacun de penser que les grands événements de l'histoire romaine - et notamment le glissement de la République au Principat - avaient valeur d'exempla, s'actualisant dans le présent de l'Angleterre guillaumienne.

\author{
Charles-Édouard LEVILLAIN \\ Institut d'Études Politiques - Université de Lille II
}

37. Pour ce sujet voir John G. A. Pocock, The Machiavellian Moment. Florentine Political Thought and the Allantic Republican Tradition (Princeton: Princeton UP, 1975); Eco Haitsma Mulier, The Myth of Venice and Dutch Republican Thought in the Seventeenth Century (Assen: Gorcum, 1980). 